\title{
Anchoring Transitions of Liquid Crystals for Optical Amplification of Phospholipid Oxidation Inhibition by Ascorbic Acid
}

\author{
Minmin Zhang and Chang-Hyun JANG ${ }^{\dagger}$ \\ Department of Chemistry, Gachon University, Seongnam-Si, Gyeonggi-Do 461-701, Korea
}

\begin{abstract}
There is considerable evidence that the antioxidant property of ascorbic acid (AH) is effective for reducing oxidative stress of phospholipids. Herein, a liquid crystals (LCs)-based method was developed for the optical amplification of resistance to phospholipid oxidation by AH. Phospholipid peroxidation initiated by free radicals was monitored from a homeotropic-to-planar anchoring transition of LCs via polarized optical microscopy. Alternatively, consistent homeotropic anchoring of LCs was observed when the oxidation caused by free radicals was blocked by $\mathrm{AH}$.
\end{abstract}

Keywords Ascorbic acid, antioxidant, phospholipids, liquid crystals, anchoring transition

(Received March 25, 2015; Accepted July 16, 2015; Published December 10, 2015)

\section{Introduction}

Phospholipids are the most abundant lipids in cell membranes. They play an important role in cellular processes by self-organizing in the membrane bilayer and, together with intrinsic membrane proteins, form a permeable barrier of cells and organelles. ${ }^{1-3}$ Oxidative degradation of phospholipids, especially those induced by reactive oxygen species (ROS), seems to be associated with many multifactorial diseases, especially cancer, cardiovascular diseases, and inflammatory disorders. ${ }^{4,5}$ ROS, such as superoxides, hydrogen peroxide $\left(\mathrm{H}_{2} \mathrm{O}_{2}\right)$, hydroxyl radicals, and singlet oxygen, are formed by a variety of enzymatic reactions..$^{6-8}$ In the present study, the hydroxyl radical initiating phospholipid peroxidation was generated by the Fenton reaction. The Fenton reaction is the oxidation of organic substrates by a mixture of $\mathrm{H}_{2} \mathrm{O}_{2}$ and ferrous iron. ${ }^{9}$ Once the $\mathrm{OH} \cdot$ is generated, the degradation or oxidation of organic compounds sequentially takes place in solution when the radicals diffuse near the surface.

Antioxidants are able to delay and/or prevent lipid oxidation in fatty systems by different mechanisms. ${ }^{10-12}$ Among natural compounds with antioxidant properties, ascorbic acid (AH) is used without restriction in foods and cosmetics for its strong antioxidant activity. ${ }^{13,14}$ Assays for identifying and characterizing the antioxidation of AH have been the subject of many studies. Yamamoto et al. used a superoxide probe of MPEC (2-methyl6- $p$-methoxyphenylethynylimidazopyrazinone) by the optical fiber method to determine the optimal amount of vitamin $\mathrm{E}$ coating necessary to improve the antioxidation property of the vitamin E-coated dialysis membrane and recovery of oxidized vitamin $\mathrm{E}$ by vitamin $\mathrm{C}$ treatment. ${ }^{7}$ Additionally, Das et al. used the colorimetric method to measure MDA (malondialdehyde), which is a marker of lipid peroxidation, to investigate the inhibition of LDL (low-density lipoprotein)

† To whom correspondence should be addressed.

E-mail: chjang4u@gachon.ac.kr oxidation by AH. ${ }^{13}$ All of these existing process-based assays are complicated, time consuming, and require sophisticated instrumentations. Despite a substantial amount of research conducted on this topic, extensive knowledge has not yet been gained regarding the power of antioxidants derived from plants, nor has their potential been substantially tapped.

We recently reported that a phospholipid monolayer that selfassembles at the aqueous/liquid crystal (LC) interface is coupled to the orientation of LCs. ${ }^{15-17}$ The biological activity involved in combining phospholipids was demonstrated to trigger orientational transition of LCs that can be readily visualized under crossed polarizers. ${ }^{18-20}$ This coupling provides the foundation for transduction and amplification of biological events that occur at these interfaces. To the best of our knowledge, most previously reported research has focused on the secondary oxidation products or decomposition products of phospholipids. LCs have never been used to study phospholipid oxidation at the aqueous-LC interface or for determining the effect of naturally occurring antioxidants, especially $\mathrm{AH}$, on this oxidation. In the present study, we undertook an LC-based optical and kinetic study to evaluate the oxidation of polyunsaturated fatty acids that occurs at the interface between the aqueous phase and immiscible liquid crystals and its inhibition of oxidation in solution by vitamin $\mathrm{C}$. The aim of this study was primarily to elucidate the rate and mechanism of lipid peroxidation and its inhibition.

\section{Experimental}

\section{Materials}

Tris buffered saline (TBS; $0.05 \mathrm{M}$ Tris, $0.138 \mathrm{M} \mathrm{NaCl}$, and $0.0027 \mathrm{M} \mathrm{KCl}, \mathrm{pH} 8.0)$, sulfuric acid, $\mathrm{H}_{2} \mathrm{O}_{2}$ (30\% w/v), octyltrichlorosilane (OTS), 2-arachidonoyl-1-palmitoyl-snglycero-3-phosphocholine (PAPC), AH, sodium chloride, hydrochloric acid, sodium hydroxide, chloroform, ethanol, methanol, methylene dichloride, and capillary tubes were purchased from Sigma-Aldrich (St. Louis, MO). LC, 4'-pentyl- 


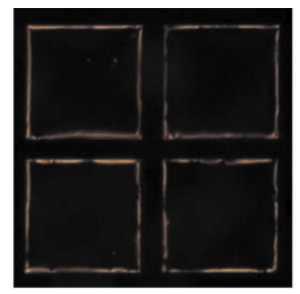

A

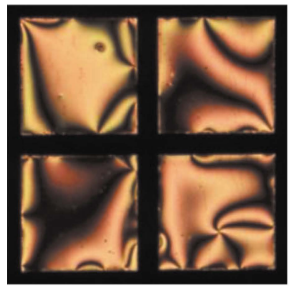

B

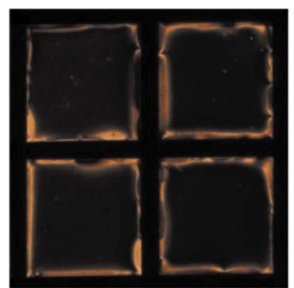

C

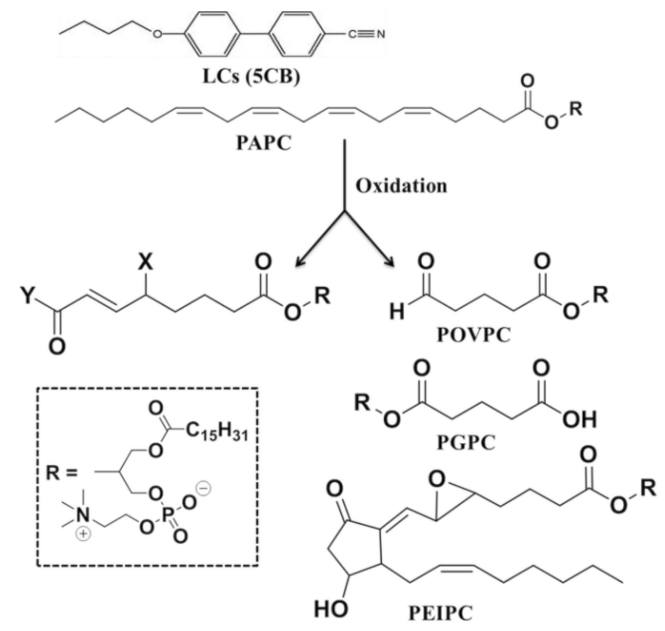

D

Fig. 1 Cross-polarized optical images of 5CB at an aqueous-LC interface after being exposed to (A) PAPC solution, (B) oxidized PAPC solution, and (C) AH-PAPC solution with Fenton reagent. (D) Representative chemical structures of LCs (5CB) and oxidized phospholipids formed during oxidation of PAPC. Here X represents hydroxyl $(\mathrm{OH})$ or ketone $(=\mathrm{O})$, Y represents hydroxyl $(\mathrm{OH})$ or hydrogen $(\mathrm{H})$.

4-cyanobiphenyl (5CB), was purchased from EM Industries (Hawthorne, NY). Copper specimen grids (50 meshes, $500 \mu \mathrm{m}$ pitch, $420 \mu \mathrm{m}$ hole, $80 \mu \mathrm{m}$ bar, $25 \pm 5 \mu \mathrm{m}$ thickness) were obtained from GILDER GRIDS (Grantham, Lincs). Premium glass microscope slides were purchased from Fisher Scientific (Pittsburgh, PA). Ultrapure water, with a resistivity of $18.2 \mathrm{M} \Omega \mathrm{cm}$, was obtained from a Milli-Q system (Millipore, Bedford, MA).

\section{Treatment of glass microscope slides with OTS}

Glass slides were immersed in piranha solution $[70 \%(\mathrm{v} / \mathrm{v})$ sulfuric acid and $30 \%(\mathrm{v} / \mathrm{v}) \mathrm{H}_{2} \mathrm{O}_{2}$ ] for $30 \mathrm{~min}$ at $\sim 80^{\circ} \mathrm{C}$. The slides were then rinsed with water, ethanol, and methanol and dried under a stream of gaseous nitrogen, after which they were heated at $120^{\circ} \mathrm{C}$ overnight prior to OTS deposition. A $0.5 \mathrm{mM}$ OTS solution was prepared, and the piranha-cleaned slides were immersed in $0.5 \mathrm{mM}$ OTS in a heptane solution and allowed to stay at room temperature for $30 \mathrm{~min}$. The samples were then rinsed with methylene dichloride and dried under nitrogen.

\section{Formation of AH-PAPC vesicles}

AH-TBS solution was obtained by dissolving solid $\mathrm{AH}$ in TBS buffer (TBS consisting of $0.05 \mathrm{M}$ Tris and adjusted to a $\mathrm{pH}$ of 5.8 prior to use). Phospholipids dissolved in chloroform $(50 \mathrm{mg} / \mathrm{mL})$ were dried with $\mathrm{N}_{2}$ gas and desiccated under vacuum for at least $3 \mathrm{~h}$. Dried phospholipids were resuspended with AH-TBS to a final concentration of $1 \mathrm{mM}$. The phospholipid suspension was then sonicated three times for 5 min each to clarify using a probe sonicator, filtered twice with a $0.22-\mu \mathrm{m}$ filter, and typically used within $2 \mathrm{~d}$ after preparation.

\section{Preparation of a glass slide-supported LC optical cell}

OTS-treated glass slides were fixed to the bottom of an eight-well chamber slide with silicone mounting medium. Subsequently, transmission electron microscopy grids (50 meshes and $25 \pm 5 \mu \mathrm{m}$ thickness) were placed onto the slide. The copper grid was then impregnated with $2 \mu \mathrm{L}$ of $5 \mathrm{CB}$ using a Hamilton syringe. By heating to isotropic phase $\left(>35^{\circ} \mathrm{C}\right)$, excess $5 \mathrm{CB}$ was removed from the LCs with a $20-\mu \mathrm{L}$ capillary tube. The grids containing LCs were then immersed into the aqueous solutions of interest. Each assay was independently performed at least six times.

\section{LC-based technique for testing the antioxidation of $A H$}

Prior to the identification of the antioxidation effects of $\mathrm{AH}$ on phospholipids, $400 \mu \mathrm{L}$ of a solution containing AH-PAPC (in $0.05 \mathrm{M}$ Tris, $0.138 \mathrm{M} \mathrm{NaCl}$, and $0.0027 \mathrm{M} \mathrm{KCl}$; the $\mathrm{pH}$ was adjusted prior to use) was reacted with Fenton reagent $(5 \mu \mathrm{L}$ $\mathrm{FeSO}_{4}$ solution $(50 \mathrm{mM})$ and $500 \mu \mathrm{L}$ of hydrogen peroxide $\left.\left(\mathrm{H}_{2} \mathrm{O}_{2}\right)(25 \mathrm{mM})\right)$ for $2 \mathrm{~h}$ at room temperature with continuous shaking. Subsequently, 5CB (confined in copper grids) was immersed in these mixed solutions.

\section{Results and Discussion}

LC-based sensor for imaging oxidation effects of the Fenton reaction on bioactive phospholipids

LCs were employed for real-time and label-free characterization of phospholipid oxidation by the Fenton reaction. Figure 1D shows the representative chemical structure of LCs and oxidized phospholipids forming during Fenton reaction. 2-Arachidonoyl1-palmitoyl-sn-glycero-3-phosphocholine (PAPC), owing to its amphiphilic properties, self-assembled at the aqueous-LC interface, causing the LC optical images to turn dark and indicating a homeotropic alignment of LC molecules at the interface (Fig. 1A). This result is consistent with previous research, which demonstrated that the orientational changes of LCs correspond to the formation of the phospholipid monolayer at aqueous-LC interfaces. ${ }^{16}$ In this study, phospholipid solution at a concentration of $0.5 \mathrm{mM}$ was utilized as a detection substrate. After oxidation by Fenton reaction, oxidized PAPC which remain at the interface prevent self-assembling, inducing orientational changes of LCs from the homeotropic to planar transition. As a result, a dark-to-bright optical change was observed through polarized optical microscopy (Fig. 1B). Subsequently, the optical signals that represented the oxidation level of phospholipids at different concentrations of $\mathrm{H}_{2} \mathrm{O}_{2}$ were 


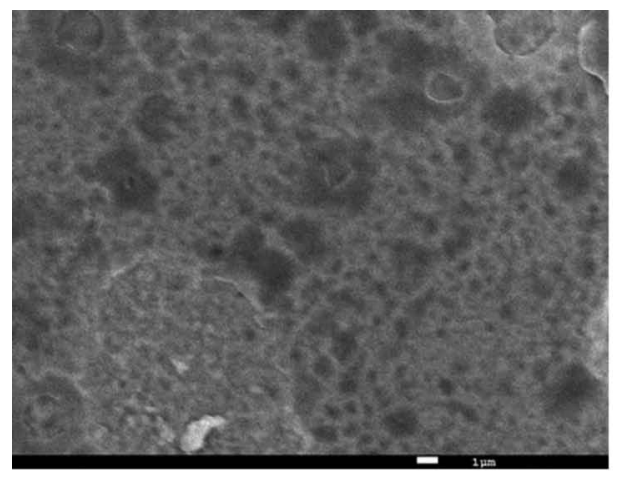

A

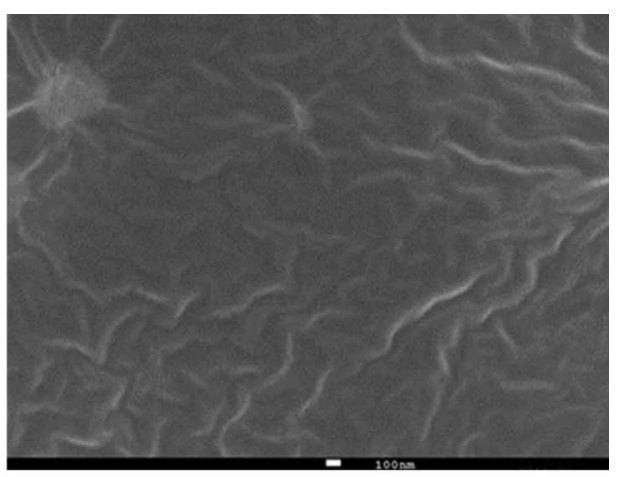

B

Fig. 2 SEM images of the PAPC monolayer under Fenton oxidation (A) without AH and (B) with AH.

observed in real time. The sensitivity of $\mathrm{H}_{2} \mathrm{O}_{2}$, a vital component of the Fenton reagent, was also determined to be $25 \mathrm{mM}$ at room temperature. ${ }^{17}$ Compared with previous research by Brandi and Baatout, ${ }^{21,22}$ the detection limit is reasonable. Brandi et al. found that cell membrane permeability was markedly increased in cell membranes at $\mathrm{H}_{2} \mathrm{O}_{2}$ concentration $>13.25$ mM. ${ }^{21}$ We believe that the sensitivity of $\mathrm{H}_{2} \mathrm{O}_{2}$ can be still increased if we increase the temperature and prolong the incubation time.

\section{Role of AH in phospholipid oxidation}

It is well-known that $\mathrm{AH}$ works as a donor antioxidant, it could be considered a valid alternative to protect lipids from oxidation. As we mentioned above, when 5CB films were exposed to phospholipids oxidized by the Fenton reaction, the optical images of 5CB changed from dark to bright colors (Figs. 1A and 1B). However, after $\mathrm{AH}$ was added into the mixed aqueous solution of PAPC and Fenton reagent and incubated for $2 \mathrm{~h}$ at room temperature with continuous shaking, $5 \mathrm{CB}$ films immersed under $400 \mu \mathrm{L}$ of this solution remained dark, indicating a homeotrapic state in LC orientation (Fig. 1C). After $1 \mathrm{~h}$ passed, the uniformly dark appearance of the LCs was still sustained. We observed the optical response for more than $4 \mathrm{~h}$, the image remained black during contact with the solution, maintaining the homeotropic alignment. These results indicate that the presence of antioxidants in the phospholipid oxidation process is an effective way to protect the lipid from oxidative damage.

We confirmed antioxidation of AH by field emission scanning electron microscopy (FE-SEM). Figure 2 shows SEM images of the PAPC membrane with and without the addition of $\mathrm{AH}$ prior to the initiation of Fenton oxidation. Phospholipid membrane specimens that were pre-incubated with different solutions were transferred onto a silicon wafer. After the membrane was dried overnight at room temperature, SEM images were obtained using an FE-SEM (JEOL Corp., JSM $6700 \mathrm{~F}$ ) at $15.00 \mathrm{kV}$. The SEM image shows that the phospholipid membrane has a porous morphology after oxidation by the Fenton reaction (Fig. 2A). Instead, after AH was dispersed as an aqueous solution directly in Fenton reagent, the oxidation effect of free radicals on phospholipids was substantially reduced, as shown in Fig. 2B. It is clear that the antioxidant activity derived from $\mathrm{AH}$ protected the phospholipid from being destroyed. We also used UV/Vis spectroscopy to confirm the antioxidation of $\mathrm{AH}$ on phospholipid oxidation (Fig. 3). The absorption of a conjugated diene at $235 \mathrm{~nm}$ was used to

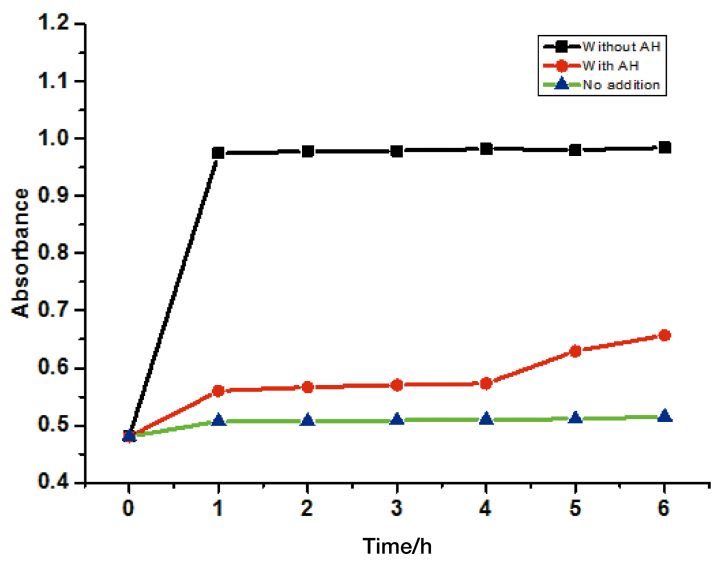

Fig. 3 Observed UV/Vis absorbance as a function of time at $235 \mathrm{~nm}$ for ( $\mathbf{\square}$ PAPC monolayer by Fenton oxidation with addition of AH, ( PAPC monolayer by Fenton oxidation without addition of $\mathrm{AH}$, and $(\mathbf{\Delta})$ PAPC solution (control). The solid lines serve to guide the eye. Values indicate the means of three repeated measurements \pm SD.

determine the concentration of hydroperoxides. ${ }^{23}$ To calculate absorbance at a given wavelength, the computer in the spectrophotometer simply takes the intensity of light at that wavelength before it passes through the sample $\left(I_{0}\right)$, divides this value by the intensity of the same wavelength after it passes through the sample $(I)$, then takes the $\log 10$ of that number:

$$
A=\log I_{0} / I
$$

The illustration in Fig. 3 reveals the peroxide value as a function of oxidation time. It is apparent that the antioxidation of $\mathrm{AH}$ inhibited phospholipid oxidation.

After we confirmed the antioxidant activity of $\mathrm{AH}$, we monitored the antioxidation of $\mathrm{AH}$ in more detail. We next studied in vitro phospholipid oxidation in the presence of different concentrations of $\mathrm{AH}$. We found that the antioxidant activity was concentration-dependent. A series of mixed solutions of PAPC pre-incubated with Fenton reagent and AH at different concentrations was introduced into the LC optical chamber. When $400 \mu \mathrm{L}$ of $500 \mu \mathrm{M}$ PAPC solution pre-incubated with Fenton reagent and $500 \mu \mathrm{M}$ AH was introduced into the 5CB film chamber, a dark appearance (Fig. 4B) was displayed as an optical response, indicating a hometropic orientation of LCs at the aqueous/LC interface. This result was comparable to 


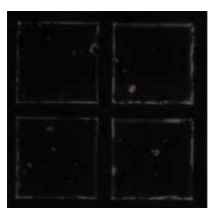

A

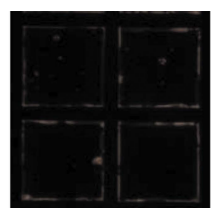

B

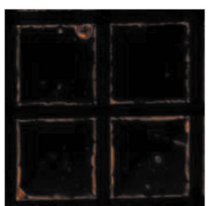

C

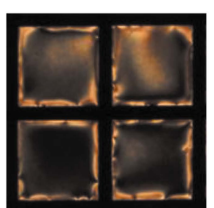

D

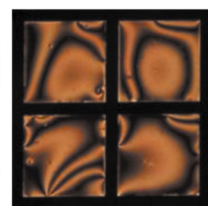

$\mathrm{E}$

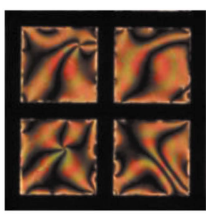

$\mathrm{F}$

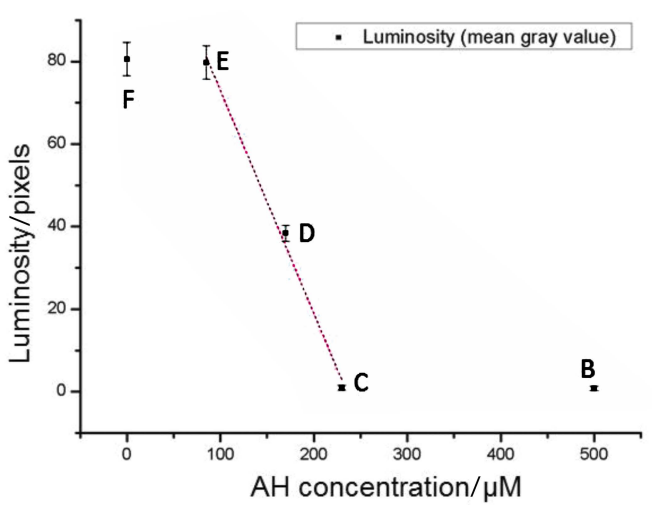

G

Fig. 4 Cross-polarized optical images of 5CB at an aqueous/LC interface after being exposed to a series of (A) AH-PAPC solutions, and AH-PAPC solutions oxidized by the Fenton reaction with the following concentrations of AH: (B) $500 \mu \mathrm{M}$, (C) $230 \mu \mathrm{M}$, (D) $170 \mu \mathrm{M}$, (E) $85 \mu \mathrm{M}$, and (F) $0 \mu \mathrm{M}$. (G) Luminosity (mean gray value) of LC images as a function of $\mathrm{AH}$ concentrations. Values indicate the means of three repeated measurements \pm SD.

the optical response of LC contact with pure PAPC solution (Fig. 4A). Next, we employed AH solution at a concentration of $230 \mu \mathrm{M}$; the same dark image of LC was obtained, suggesting a homeotropic alignment of LCs at the aqueous/LC interface (Fig. 4C). Then, we carried out the same detection using $\mathrm{AH}$ at a concentration of $170 \mu \mathrm{M}$. Contrary to the above results, the LCs adopted a partially bright appearance when they were immersed under PAPC solution that had been pre-incubated with the mixed solution of Fenton reagent and $170 \mu \mathrm{M} \mathrm{AH}$, corresponding to a tilted alignment of LCs at the aqueous/LC interface (Fig. 4D). When we lowered $\mathrm{AH}$ concentration to $85 \mu \mathrm{M}$, the LCs immediately became entirely bright in optical appearance and remained bright for $1 \mathrm{~h}$, indicating that the orientation of LC transitioned to planar anchoring (Fig. 4E). This appearance was similar to the one we obtained from PAPC solution that was solely pre-incubated with Fenton reagent (Fig. 4F), where LCs produced a bright response and indicated a planar alignment at the aqueous-LC interface. These findings strengthened the notion that there is an increase in phospholipid oxidation, which is reflected by increased luminosity in LC images. In an effort to quantify the luminosity of the LC image, we used the ImageJ software to convert images to grey scale, and the average pixel brightness of each analyzed region was calculated. The luminosity of the optical images of 5CB was evaluated after contact with PAPC solution pre-incubated with Fenton reagent and $\mathrm{AH}$ solutions at different concentrations. The dynamic response of $\mathrm{LC}$ to $\mathrm{AH}$ at different concentrations, included an inverse S-curve typical for LC-based imaging response, showing the threshold of antioxidation (Fig. 4G). From the results above, it is clear that antioxidant activity has a strongly positive correlation with AH concentration.

\section{Conclusions}

In summary, we demonstrated an LC-based detection technique for label-free characterization of phospholipid oxidation by the Fenton reaction. Phospholipid oxidation disrupted the integrity and organization of the lipid membrane and induced a bright appearance in the aqueous-LC interface, and $\mathrm{AH}$ was used as an effective antioxidant to protect the lipid from oxidative damage. After the addition of AH solution, the optical response reverted back to a dark appearance, indicating that the phospholipid monolayer formed at the aqueous-LC interface, prior to the oxidation. Moreover, the results suggest that $\mathrm{AH}$ showed concentration-dependent activity.

\section{Acknowledgements}

This study was supported by a grant from the Korean Health Technology R\&D Project, Ministry of Health \& Welfare, Republic of Korea (HI13C0891) and Basic Science Research Program through the National Research Foundation of Korea (NRF) funded by the Ministry of Science, ICT \& Future Planning (NRF-2013R1A1A1A05008333).

\section{References}

1. C. Striebel, A. Brecht, and G. Gauglitz, Biosens. Bioelectron., 1994, 9, 139.

2. V. B. O'Donnell and R. C. Murphy, Blood, 2012, 120, 1985.

3. S. Heyse, H. Vogel, M. Sänger, and H. Sigrist, Protein Sci., 1995, 4, 2532.

4. B. Halliwell and J. Gutteridge, "Free radicals in biology 
and medicine", 1985, Pergamon, New York.

5. P. M. Abujaa and R. Albertini, Clin. Chim. Acta, 2001, 306, 1.

6. S. Choksi, Y. Lin, Y. Pobezinskaya, L. Chen, C. Park, M. Morgan, T. Li, S. Jitkaew, X. Cao, Y. S. Kim, H. S. Kim, P. Levitt, G. Shih, M. Birre, C. X. Deng, and Z. G. Liu, Mol. Cell., 2011, 42, 597.

7. K. Yamamoto, M. Matsuda, M. Okuoka, T. Yakushiji, M. Fukuda, T. Miyasaka, Y. Matsumoto, and K. Sakai, J. Membr. Sci., 2007, 302, 115.

8. H.-P. Deigner and A. Hermetter, Curr. Opin. Lipidol., 2008, 19, 289.

9. H. B. Dunford, Coord. Chem. Rev., 2002, 233 - 234, 311.

10. M. Laguerre, J. Lecomte, and P. Villeneuve, Prog. Lipid Res., 2007, 46, 244.

11. M. Mosca, A. Ceglie, and L. Ambrosone, J. Phys. Chem. B, 2008, 112, 4635 .

12. D. Steinberg, Circulation, 1992, 85, 2337.

13. S. Das, Snehlata, N. Das, and L. M. Srivastava, Clin. Chim.
Acta, 2006, 372, 202.

14. Z.-S. Jia, B. Zhou, L. Yang, L.-M. Wu, and Z.-L. Liu, J. Chem. Soc., Perkin Trans., 1998, 2, 911.

15. Q. Z. Hu and C. H. Jang, Analyst, 2012, 137, 567.

16. M. Zhang and C. H. Jang, Anal. Biochem., 2014, 455, 13.

17. M. Zhang and C. H. Jang, J. Biosci. Bioeng., doi: 10.1016/j. jbiosc.2014.12.016.

18. J.-S. Park, S. Teren, W. H. Tepp, D. J. Beebe, E. A. Johnson, and N. L. Abbott, Chem. Mater., 2006, 18, 6147.

19. J. M. Brake, M. K. Daschner, Y. Y. Luk, and N. L. Abbott, Science, 2003, 302, 2094.

20. J.-S. Park and N. L. Abbott, Adv. Mater, 2008, 20, 1185.

21. G. Brandi, L. Salvaggio, F. Cattabeni, O. Cantoni, and K. E. Mortelmans, Environ. Mol. Mutagen., 1991, 18, 22.

22. S. Baatout, P. De Boever, and M. Mergeay, Appl. Biochem. Microbiol., 2006, 42, 369.

23. M. Kawakatsu, J. Terao, and S. Matsushita, Agric. Biol. Chem., 1984, 48, 1275. 